\title{
Preoperative liver functional volumetry performed by 3D-99mTc-GSA scintigraphy/vascular fusion imaging using SYNAPSE VINCENT: a preliminary study
}

\author{
Hiroshi Yoshida ${ }^{1}$, Hiroshi Makino, Tadashi Yokoyama', Hiroshi Maruyama1, Atsushi \\ Hirakata' ${ }^{1}$ Junji Ueda' ${ }^{1}$ Yasuhiro Mamada², Nobuhiko Taniai ${ }^{2}$, Eiji Uchida²
}

${ }^{1}$ Department of Surgery, Nippon Medical School Tama Nagayama Hospital, Tokyo 206-8512, Japan.

${ }^{2}$ Department of Surgery, Nippon Medical School, Tokyo 113-8603, Japan.

\begin{abstract}
Aim: The present study was designed to evaluate the feasibility of preoperative liver functional volumetry performed by 3D-technetium-99m-diethylenetriaminepentaacetic acid-galactosyl-human serum albumin (99mTc-GSA) scintigraphy/ vascular fusion imaging using SYNAPSE VINCENT and to examine the discrepancy between conventional and functional volumetry. Methods: The study group comprised 15 patients who underwent preoperative 3-dimensional (3D)-99mTc-GSA scintigraphy/vascular fusion imaging using SYNAPSE VINCENT software before hepatectomy between July 2014 and August 2015. The diagnosis was hepatocellular carcinoma $(n=4)$, metastatic liver tumor ( $n=$ 10), or intrahepatic cholangiocarcinoma $(n=1)$. Right hepatectomy was performed in 2 patients, left hepatectomy in 3 patients, right posterior sectionectomy in 3 patients, segmentectomy in 2 patients, and partial hepatectomy in 4 patients. 99mTc-GSA scintigraphy and computed tomography (CT) were performed to construct 3D-99mTc-GSA scintigraphy/ vascular fused images. The conventional volume ratio of the planned resection region without tumor (\% CT), and the functional volume ratio of the planned resection region without tumor ( $\% \mathrm{GSA}$ ) were calculated. The discrepancy ratio was calculated as follows: discrepancy ratio $=100-\%$ GSA $\%$ CT $\times 100$ (\%). Results: The $\%$ GSA $(17.9 \pm 16.7 \%)$ was significantly lower than the \% CT $(21.5 \pm 17.6 \%)(P<0.036)$. In all except 2 patients, the $\%$ GSA was lower than the $\%$ CT. The discrepancy ratio ranged from $-4 \%$ to $75 \%$ (median, 20.7\%). Conclusion: 3D-99mTc-GSA scintigraphy/ vascular fused images constructed using SYNAPSE VINCENT were useful for noninvasively performing functional liver volumetry in patients scheduled to undergo various patterns of hepatectomy. In planned resection regions without tumor, the functional volume ratio was about $20 \%$ lower than the conventional volume ratio.
\end{abstract}

Key words: Functional volumetry; 99m-diethylenetriaminepentaacetic acid-galactosyl-human serum albumin; SYNAPSE VINCENT; fusion image; 3-dimensional; computed tomography

Address for correspondence:

Dr. Hiroshi Yoshida, Department of Surgery, Nippon Medical School Tama Nagayama Hospital, 1-7-1 Nagayama, Tama-city, Tokyo 206-8512, Japan. E-mail: hiroshiy@nms.ac.jp

Received: 25-03-2016, Accepted: 29-03-2016

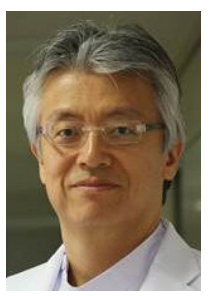

Hiroshi Yoshida, M.D., is graduated from Nippon Medical School (Bsc) obtained MD and subsequently entered the Department of Surgery in 1986. From 1988 to 1992, He attended Postgraduate course in Department of Surgery, Nippon Medical School and obtained PhD. In 2003, he was appointed assistant professor and, in 2005, associated professor of the Department of Surgery, Nippon Medical School. In 2011, he was appointed Chief and Clinical Professor of the Department of Surgery, Nippon Medical School Tama Nagayama Hospital. In 2016, he was appointed Director of the Nippon Medical School Tama Nagayama Hospital.

\begin{tabular}{|l|c|}
\hline \multicolumn{3}{|c|}{ Access this article online } \\
\hline \multirow{2}{*}{ Website: } & Quick Response Code \\
http://www.hrjournal.net/ & \\
\hline DOI: &
\end{tabular}

This is an open access article distributed under the terms of the Creative Commons Attribution-NonCommercial-ShareAlike 3.0 License, which allows others to remix, tweak, and build upon the worknon-commercially, as long as the author is credited and the new creations are licensed under the identical terms.

For reprints contact: service@oaepublish.com

How to cite this article: Yoshida $\mathrm{H}$, Makino $\mathrm{H}$, Yokoyama $\mathrm{T}$, Maruyama H, Hirakata A, Ueda J, Mamada Y, Taniai N, Uchida E. Preoperative liver functional volumetry performed by 3D-99mTc-GSA scintigraphy/ vascular fusion imaging using SYNAPSE VINCENT: a preliminary study. Hepatoma Res 2016;2:187-92. 


\section{INTRODUCTION}

In liver surgery, preoperative treatment planning is defined in the context of the anatomical structure and the functional reserve of the liver. In patients who have damaged liver function or who are scheduled to undergo extended hepatectomy, the postoperative residual liver volume with adequate preservation of blood supply and drainage vessels is very important for the prevention of liver failure. ${ }^{[1]}$

Virtual reality simulation on computed tomography (CT), magnetic resonance imaging (MRI), or ultrasonography (US) plays an important role in examining the anatomical structure of the liver. Recently 3-dimensional (3D) imaging techniques, such as 3D CT, 3D MRI, and 3D US, have been developed. To date, a number of methods and software systems have been developed for 3D surgical planning before liver surgery. ${ }^{[2-6]}$ Remnant liver volume can thus be determined (volumetry) before hepatectomy.

Technetium-99m-diethylenetriaminepentaacetic acidgalactosyl-human serum albumin $\left.{ }^{99 \mathrm{~m}} \mathrm{Tc}-\mathrm{GSA}\right)$ is an analog ligand of asialoglycoprotein. ${ }^{99 m} \mathrm{Tc}-\mathrm{GSA}$ binds to the asialoglycoprotein receptor (ASGP-R) located specifically on hepatocytes. The ASGP-Rconcentration is helpful in evaluating the extent and progression of liver disease, so the hepatic uptake of ${ }^{99 m} \mathrm{Tc}-\mathrm{GSA}$ reflects the number of functioning hepatocytes. ${ }^{[7-11]}$ Before hepatic resection, however, it is difficult to correctly estimate the functional hepatocyte mass of the remnant liver.

Thus, ${ }^{99 m}$ Tc-GSA scintigraphy combined with single-photo emission computerized tomography (SPECT) and CT fused imaging has been used to estimate the future remnant liver function before hepatic resection. ${ }^{[12-16]}$ However, the planned resection region had to be set manually using a 2-dimensional CT display. It was difficult to estimate the local remnant liver function in detail.

The volume analyzer software SYNAPSE VINCENT (Fujifilm Medical Co., Tokyo, Japan) is a high-speed 3D image analysis system. Using previously captured CT or MRI, high-definition 3D images of organs and vessels can be reconstructed quickly. ${ }^{[4]}$ It has become easy to grasp the complex anatomical relations between the portal triad, hepatic veins, and local tumor by volume rendering. With this software, the surgeon can simulate various patterns of planned hepatectomy. ${ }^{[4-6]}$

In the present study, we performed preoperative liver functional volumetry by 3D-99mTc-GSA scintigraphy/ vascular fused imaging using SYNAPSE VINCENT and examined the discrepancy between conventional and functional volumetry.

\section{METHODS}

This retrospective study was approved by our institutional review board; informed consent was obtained from all patients before ${ }^{99 \mathrm{~m}} \mathrm{Tc}-\mathrm{GSA}$ scintigraphy and CT. We retrospectively reviewed patients who had undergone liver surgery between July 2014 and August 2015 in the Department of Surgery of our hospital. Twenty-five patients preoperatively underwent 3D-99m Tc-GSA scintigraphy/ vascular fused imaging using SYNAPSE VINCENT.

The following exclusion criteria were applied: (1) a history of hepatectomy or portal embolization; (2) hilar cholangiocarcinoma with unilateral biliary drainage; and (3) hepatectomy for benign disease. A total of 15 patients (10 men and 5 women; age, 60 to 81 years; mean, 72.7 years) who agreed to undergo preoperative 3D- ${ }^{99 \mathrm{~m}} \mathrm{Tc}-\mathrm{GSA}$ scintigraphy/vascular imaging using SYNAPSE VINCENT were studied. The diagnosis was hepatocellular carcinoma in 4 patients, metastatic liver tumor in 10 patients, and intrahepatic cholangiocarcinoma in 1 patient. Based on the Brisbane 2000 classification criteria, ${ }^{[17]}$ right hepatectomy was performed in 2 patients, left hepatectomy in 3 patients, right posterior sectionectomy in 3 patients, segmentectomy in 2 patients, and partial hepatectomy in 5 patients. Background of the liver was liver cirrhosis in 2 patients, chronic hepatitis in 1 patient, and normal liver in 12 patients. The planned resection region of the liver could be similarly resected in all patients.

\section{D-vascular imaging using SYNAPSE VINCENT}

Preoperative enhanced CT was performed with a 64-multidetector-row CT scanner (Discovery CT 750 HD, GE Healthcare Japan, Co. Ltd., Tokyo, Japan) at $0.625-\mathrm{mm}$ intervals. Four-phase contrast-enhanced CT was performed $30,60,90$, and $180 \mathrm{~s}$ after initiating the injection of contrast media to obtain hepatic arterial, portal venous, hepatic venous, and equilibrium phase images, respectively. A total of $100 \mathrm{~mL}$ of nonionic contrast material containing $370 \mathrm{mg}$ of iodine per milliliter (Iopamidol, Bayer Yakuhin, Osaka, Japan) was injected intravenously at a rate of $3.3 \mathrm{~mL} / \mathrm{s}$ using an automatic power injector. With the use of a workstation, a routine preoperative CT workup was performed in the axial and coronal imaging planes. The data were obtained in Digital Imaging and Communications in Medicine format and transmitted to a workstation running SYNAPSE VINCENT.

Liver Analysis Application uses Dynamic-CT imaging of the liver. After data for the hepatic arterial, portal venous, and hepatic venous phases are obtained, operative simulation by 3D-vascular images is performed. After selection of the portal venous branch of the planned resection area, surgical simulations can be displayed. This system also can calculate the total liver volume, tumor volume, and volume of planned resection region. ${ }^{[4]}$

\section{${ }^{99 m}$ Tc-GSA scintigraphy}

A bolus of $1 \mathrm{~mL}$ of ${ }^{99 \mathrm{~m} T c-G S A}$ (185 MBq, Nihon Medi-physics Co. Ltd., Nishinomiya, Japan) was intravenously injected 
into an antecubital vein. Images were obtained with the patient in the supine position, using a gamma camera over a large field of view in which a high-resolution, allpurpose parallel-hole collimator (Infinia: GE Healthcare Japan Co. Ltd., Tokyo, Japan) was centered over the liver and precordium. Computer acquisition of gamma camera data was initiated simultaneously with injection of ${ }^{99 \mathrm{~m}} \mathrm{Tc}-\mathrm{GSA}$ and stopped at $30 \mathrm{~min}$. Digital images (128 $\times 128$ pixels) were acquired in the byte mode at a rate of 2 frames/min for $20 \mathrm{~min}$ after the injection. Hepatic SPECT data were obtained for $15 \mathrm{~min}$ after the end of the dynamic scintigraphic study.

\section{D-99mTc-GSA scintigraphy/vascular fusion imaging using SYNAPSE VINCENT}

Data obtained by ${ }^{99 \mathrm{~m} T C-G S A}$ scintigraphy and CT imaging are composited by adjusting the axial and coronal images, and 3D-99m Tc-GSA scintigraphy/vascular fused images are constructed. The 3D-vascular images are used to select the portal venous branch to be resected and to calculate the extraction volume and ratio of the dominant region of the branch (conventional volumetry). In 3D-99m TcGSA scintigraphy, the functional volume and ratio of the same region are calculated (functional volumetry) concomitantly. Count-rates are displayed on the images, which can be saved. ${ }^{[4]}$

\section{Image analysis}

In ${ }^{99 m}$ Tc-GSA scintigraphy, regions of interest (ROI) over the entire liver and heart are delineated. Time-activity curves are generated for the ROI.

An index of clearance of ${ }^{99 \mathrm{~m}} \mathrm{Tc}-\mathrm{GSA}$ from the blood is calculated as the quotient of the radioactivity of the heart ROI 15 min after injection (H15) divided by the radioactivity of the heart ROI 3 min after injection (H3), $(\mathrm{HH} 15=\mathrm{H} 15 / \mathrm{H} 3)$. Hepatic uptake ratio of ${ }^{99 \mathrm{~m} T \mathrm{Tc}-G S A}$ is calculated by dividing the radioactivity of the liver ROI at 15 min (L15) by the sum of H15 and L15 (LHL15) =L15/ $[\mathrm{H} 15+\mathrm{L} 15]^{\mid 10,11,18]}$

The 3D-99mTc-GSA scintigraphy/vascular fusion images obtained using SYNAPSE VINCENT are used to calculate the total liver volume without tumor, the conventional volume ratio of the planned resection region without tumor $(\% \mathrm{CT})$, and the functional volume ratio of the planned resection region without tumor (\% GSA). The discrepancy ratio is calculated as follows: discrepancy ratio $=100-\% \mathrm{GSA} \% \mathrm{CT}$ $\times 100(\%)$.

\section{Case 9}

A 71-year-old woman was admitted with a diagnosis of hepatocellular carcinoma concomitant with liver cirrhosis due to autoimmune hepatitis. CT revealed hepatocellular carcinoma (hypervascular tumor) in Segment 6 [Figure 1]. The \% CT and \% GSA were $3.0 \%$ and $2.2 \%$ in partial hepatectomy, $12.2 \%$ and $11.7 \%$ in segmentectomy, $27.5 \%$ and $28.4 \%$ in right posterior sectionectomy, and $63.4 \%$ and $68.5 \%$ in right hepatectomy, respectively. Segmentectomy was performed to treat the hepatocellular carcinoma.

\section{Statistical analysis}

Values are expressed as means \pm standard deviation (range). The Student's $t$ test was used to compare differences between two groups. A $P$ value of $<0.05$ was significant. All analyses were performed with SPSS version 17 (SPSS, Chicago, IL).

\section{RESULTS}

The clinical characteristics of the 15 patients are summarized in Table 1. The mean values of HH15 and LHL15 were $0.64 \pm 0.10$ and $0.90 \pm 0.06$, respectively. The \% GSA $(17.9 \% \pm 16.7 \%)$ was significantly lower than the $\%$ CT $(21.5 \% \pm 17.6 \%)(P<0.036)$. In all except 2 patients, the $\% \mathrm{GSA}$ was lower than the $\% \mathrm{CT}$. The discrepancy ratio ranged from $-4 \%$ to $75 \%$ (median, $20.7 \%$ ).

\section{DISCUSSION}

Our study demonstrated that 3D-99mTc-GSA scintigraphy/ vascular fusion imaging using SYNAPSE VINCENT is useful for noninvasive functional liver volumetry in patients scheduled to undergo various patterns of hepatectomy.

Postoperative liver failure remains a life-threatening complication after hepatectomy. Conventionally, traditional liver function tests and CT volumetry have been used to evaluate patients before hepatic surgery.

The use of ${ }^{99 m}$ Tc-GSA scintigraphy to evaluate liver function was initially introduced by investigators in Japan. ${ }^{99 m} \mathrm{mc}-G S A$ is binds to the ASGP-R located specifically on hepatocytes. The function of ASGP-R remains normal even in regenerating hepatocytes, and the ASGP-R density per hepatocyte is constant. Therefore the total amounts of ASGP-R are lower in cirrhotic liver patients than in normal liver patients according to the hepatocyte theory. ${ }^{[19-23]}$ We previously reported that, with progression of hepatic functional degeneration, ASGP-R density per hepatic volume decreases, especially in the right lobe. ${ }^{[9]}$ The hepatic accumulation of ${ }^{99 \mathrm{~m}} \mathrm{Tc}-\mathrm{GSA}$ thus reflects the functional liver volume. ${ }^{[24]}$ The hepatic uptake image of ${ }^{99 \mathrm{~m}} \mathrm{Tc}-\mathrm{GSA}$ at $15 \mathrm{~min}$ or later reflects the ASGP-R population. ${ }^{|25|} \mathrm{An}$ index of clearance of ${ }^{99 \mathrm{~m} T \mathrm{Tc}-G S A}$ (HH15) is calculated as the quotient of the radioactivity of the heart ROI 15 min after injection (H15) divided by the radioactivity of the heart ROI 3 min after injection (H3), (HH15 = H15/H3). Hepatic uptake ratio of ${ }^{99 m} \mathrm{Tc}-$ GSA (LHL15) is calculated by dividing the radioactivity of the liver ROI at 15 min (L15) by the sum of H15 and L15 $(\mathrm{LHL} 15=\mathrm{L} 15 /[\mathrm{H} 15+\mathrm{L} 15]) \cdot{ }^{\mid 10,11,18]} \mathrm{HH} 15$ and LHL15 reflect the hepatic function. Various studies of ${ }^{99 \mathrm{~m}} \mathrm{Tc}$-GSA have examined hepatic function. . $^{\mid 9,10,26-28]}$ 

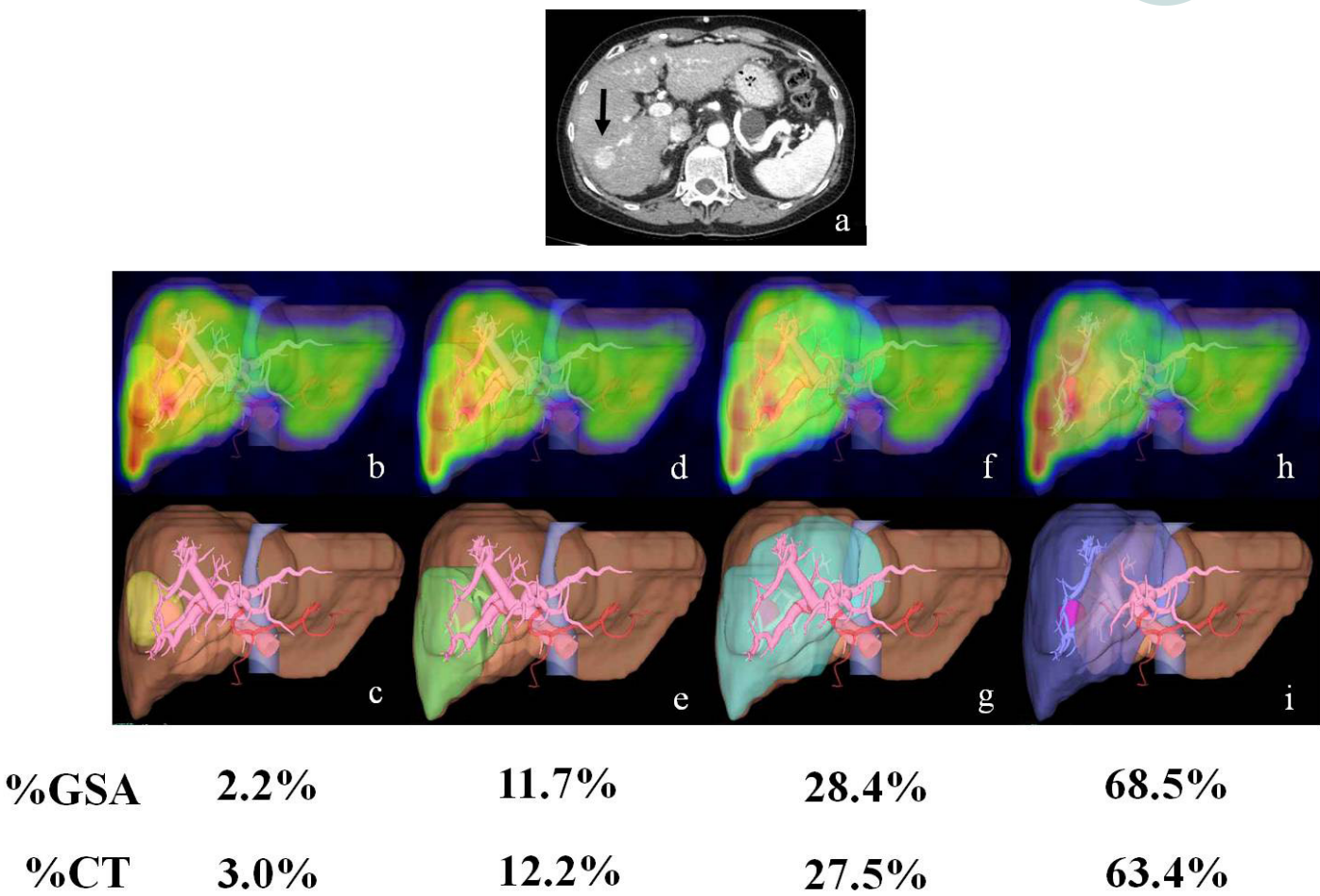

Figure 1: Various patterns of \% GSA and \% CT on 3D-99mTc-GSA scintigraphy/vascular fusion images in case 11. (a) CT image (planned resection region); (b) partial hepatectomy; (c) partial hepatectomy; (d) segmentectomy (S6); (e) segmentectomy (S6); (f) right lateral sectionectomy; (g) right lateral sectionectomy; (h) right hepatectomy on 3D-99mTc-GSA scintigraphic image; (i) right hepatectomy on 3D-vascular image. \% CT: conventional volume ratio of planned resection region without tumor; \% GSA: functional volume ratio of planned resection region without tumor

Table 1: Discrepancy ratio between \% CT and \% GSA

\begin{tabular}{|c|c|c|c|c|c|c|c|c|c|c|}
\hline Case & Age & Gender & Disease & Liver & Operation & HH15 & LHL15 & $\%$ CT & $\%$ GSA & Discrepancy ratio\% \\
\hline 1 & 60 & $\mathrm{M}$ & $\mathrm{HCC}$ & $\mathrm{CH}$ & Right hepatectomy & 0.71 & 0.84 & 33.2 & 33.7 & -2 \\
\hline 2 & 76 & M & Meta & Normal & Right hepatectomy & 0.74 & 0.94 & 66.3 & 66.4 & 0 \\
\hline 3 & 72 & M & $\mathrm{HCC}$ & Normal & Left hepatectomy & 0.44 & 0.94 & 42.7 & 19.0 & 56 \\
\hline 4 & 65 & $\mathrm{~F}$ & ICC & Normal & Left hepatectomy & 0.56 & 0.92 & 21.6 & 12.5 & 42 \\
\hline 5 & 78 & $\mathrm{~F}$ & Meta & Normal & Left hepatectomy & 0.62 & 0.90 & 32.9 & 29.1 & 12 \\
\hline 6 & 68 & M & Meta & Normal & Right posterior sectionectomy & 0.57 & 0.96 & 28.9 & 25.5 & 12 \\
\hline 7 & 74 & M & Meta & Normal & Right posterior sectionectomy & 0.70 & 0.86 & 27.5 & 23.5 & 15 \\
\hline 8 & 77 & M & Meta & Normal & Right posterior sectionectomy & 0.70 & 0.92 & 25.5 & 23.2 & 9 \\
\hline 9 & 71 & $\mathrm{~F}$ & $\mathrm{HCC}$ & LC & Segmentectomy (S6) & 0.74 & 0.88 & 12.2 & 11.7 & 4 \\
\hline 10 & 77 & M & Meta & Normal & Segmentectomy (S8) & 0.61 & 0.92 & 17.0 & 12.0 & 29 \\
\hline 11 & 81 & $\mathrm{~F}$ & $\mathrm{HCC}$ & LC & Partial hepatectom (S8) & 0.88 & 0.72 & 6.6 & 5.1 & 23 \\
\hline 12 & 77 & $\mathrm{~F}$ & Meta & Normal & Partial hepatectomy (S6) & 0.56 & 0.96 & 1.3 & 1.1 & 15 \\
\hline 13 & 78 & M & Meta & Normal & Partial hepatectomy (S6) & 0.54 & 0.94 & 1.6 & 0.4 & 75 \\
\hline 14 & 69 & M & Meta & Normal & Partial hepatectomy (S8) & 0.60 & 0.92 & 2.6 & 2.7 & -4 \\
\hline 15 & 68 & $\mathrm{M}$ & Meta & Normal & Partial hepatectomy (S8) & 0.68 & 0.88 & 2.9 & 2.2 & 24 \\
\hline $\mathrm{AV}$ & 72.7 & & & & & 0.64 & 0.90 & 21.5 & 17.9 & 20.7 \\
\hline SD & 5.6 & & & & & 0.10 & 0.06 & 17.6 & 16.7 & 21.4 \\
\hline
\end{tabular}

HCC: hepatocellular carcinoma; Meta: metastatic liver tumor; ICC: intrahepatic cholangiocarcinoma; LC: liver cirrhosis; CH: chronic hepatitis; \% CT: conventional volume ratio of planned resection region without tumor; \% GSA: functional volume ratio of planned resection region without tumor; Discrepancy ratio: (1-\% GSA \% CT) × 100; AV: average value; SD: standard deviation

The SYNAPSE VINCENT system has already been tested in various surgical fields, ${ }^{[29-32]}$ and techniques for its use have been refined. The system has been greatly helpful in previsualizing intraoperative scenarios. In fact, the time required for intraoperative confirmatory US was considerably shortened after the introduction of this technology. This system is relatively easy to operate, allowing even a novice user to create 3D images.

3D- ${ }^{99 m}$ Tc-GSA scintigraphy/vascular fusion images could also be easily created with the use of SYNAPSE VINCENT. It was possible to select the portal venous branch to be resected on the $3 \mathrm{D}$-vascular images, and the conventional volume could be compared with the functional volume of the planned resection region of the liver. The \% GSA and $\%$ CT could also be calculated and compared.

In conclusion, 3D-99m Tc-GSA scintigraphy/vascular fusion imaging performed with the use of SYNAPSE VINCENT is useful for non-invasive functional liver volumetry in patients scheduled to undergo various patterns of planned hepatectomy. There was a discrepancy between the results of conventional and functional volumetry. In the planned resection region without tumor, the functional volume ratio estimated with SYNAPSE VINCENT was about 20\% lower than the conventional 
volume ratio.

\section{Acknowledgments}

The authors thank Mr. Taisuke Sawada, Mr. Hiroshi Oda, radiological technologists, and Dr. Akira Yamamoto from Department of Radiology, Nippon Medical School Tama Nagayama Hospital, for their support in using SYNAPSE VINCENT.

\section{Financial support and sponsorship} Nil.

\section{Conflicts of interest}

There are no conflicts of interest.

\section{REFERENCES}

1. Imura S, Shimada M, Utsunomiya T. Recent advances in estimating hepatic functional reserve in patients with chronic liver damage. Hepatol Res 2015;45:10-9.

2. Conversano F, Franchini R, Demitri C, Massoptier L, Montagne F, Maffezzoli A, Malvasi A, Casciaro S. Hepatic vessel segmentation for 3D planning of liver surgery experimental evaluation of a new fully automatic algorithm. Acad Radiol 2011;18:461-70.

3. Radtke A, Sotiropoulos GC, Molmenti EP, Schroeder T, Peitgen HO, Frilling A, Broering DC, Broelsch CE, Malago' M. Computerassisted surgery planning for complex liver resections: when is it helpful? A single-center experience over an 8-year period. Ann Surg 2010;252:876-83

4. Ohshima S. Volume analyzer SYNAPSE VINCENT for liver analysis. $J$ Hepatobiliary Pancreat Sci 2014;21:235-8.

5. Ogawa C, Minami Y, Morioka Y, Noda A, Arasawa S, Izuta M, Kubo A, Matsunaka T, Tamaki N,Shibatouge M, Kudo M. Virtual sonography for novice sonographers: usefulness of SYNAPSE VINCENT ${ }^{\circledR}$ with pre-check imaging of tumor location. Oncology 2014;87 Suppl 1:50-4.

6. Shimizu T, Taniai N, Yoshioka M, Takata H, Kanda T, Mizuguchi Y, Mamada Y, Yoshida H, Uchida E. Preoperative three-dimensional virtual simulation for safe liver surgery. J Nippon Med Sch 2014;81:354-5.

7. Ashwell G, Steer CJ. Hepatic recognition and catabolism of serum glycoproteins. JAMA 1981;246:2358-64.

8. Stockert RJ, Morell AG. Hepatic binding protein: the galactosespecific receptor of mammalian hepatocytes. Hepatology 1983;3:750-7.

9. Matsuzaki S, Onda M, Tajiri T, Kim DY. Hepatic lobar differences in progression of chronic liver disease: correlation of asialoglycoprotein scintigraphy and hepatic functional reserve. Hepatology 1997;25:828-32.

10. Kokudo N, Vera DR, Makuuchi M. Clinical application of TcGSA. Nucl Med Biol 2003;30:845-9.

11. Sasaki N, Shiomi S, Iwata Y, Nishiguchi S, Kuroki T, Kawabe J, Ochi H. Clinical usefulness of scintigraphy with $99 \mathrm{mTc}$-galactosylhuman serum albumin for prognosis of cirrhosis of the liver. $\mathrm{J} \mathrm{Nucl}$ Med 1999;40:1652-6.

12. Nanashima A, Yamaguchi H, Shibasaki S, Morino S, Ide N, Takeshita H, Tsuji T, Sawai T, Nakagoe T, Nagayasu T, Ogawa Y. Relationship between CT volumetry and functional liver volume using technetium-99m galactosyl serum albumin scintigraphy in patients undergoing preoperative portal vein embolization before major hepatectomy: a preliminary study. Dig Dis Sci 2006;51:11905 .

13. Beppu T, Hayashi H, Okabe H, Masuda T, Mima K, Otao R, Chikamoto A, Doi K, Ishiko T, Takamori H,Yoshida M, Shiraishi
S, Yamashita Y, Baba H. Liver functional volumetry for portal vein embolization using a newly developed 99mTc-galactosyl human serum albumin scintigraphy SPECT-computed tomography fusion system. J Gastroenterol 2011;46:938-43.

14. Sumiyoshi T, Shima Y, Okabayashi T, Noda Y, Hata Y, Murata Y, Kozuki A, Tokumaru T, Nakamura T, Uka K. Functional discrepancy between two liver lobes after hemilobe biliary drainage in patients with jaundice and bile duct cancer: an appraisal using (99m)TcGSA SPECT/CT fusion imaging. Radiology 2014;273:444-51.

15. Yoshida M, Beppu T, Shiraishi S, Tsuda N, Sakamoto F, Okabe H, Hayashi H, Baba H,Yamashita Y. (99m)Tc-GSA SPECT/CT fused images for assessment of hepatic function and hepatectomy planning. Ann Transl Med 2015;3:17.

16. Mao Y, Du S, Ba J, Li F, Yang H, Lu X, Sang X, Li S, Che L, Tong J, Xu Y, Xu H, Zhao H, Chi T, Liu F, Du Y, Zhang X, Wang X, Dong J, Zhong S, Huang J, Yu Y, Wang J. Using Dynamic 99mT c-GSA SPECT/CT fusion images for hepatectomy planning and postoperative liver failure prediction. Ann Surg Oncol 2015;22:1301-7.

17. Strasberg SM. Nomenclature of hepatic anatomy and resections: a review of the Brisbane 2000 system. J Hepatobiliary Pancreat Surg 2005;12:351-5

18. Kwon AH, Ha-Kawa SK, Uetsuji S, Kamiyama Y, Tanaka Y. Use of technetium $99 \mathrm{~m}$ diethylenetriamine-pentaacetic acid-galactosylhuman serum albumin liver scintigraphy in the evaluation of preoperative and postoperative hepatic functional reserve for hepatectomy. Surgery 1995;117:429-34.

19. Wood AJ, Villeneuve JP, Branch RA, Rogers LW, Shand DG. Intact hepatocyte theory of impaired drug metabolism in experimental cirrhosis in the rat. Gastroenterology 1979;76:1358-62.

20. Reichen J, Arts B, Schafroth U, Zimmermann A, Zeltner B, Zysset $\mathrm{T}$. Aminopyrine $\mathrm{N}$-demethylation by rats with liver cirrhosis. Evidence for the intact cell hypothesis. A morphometric-functional study. Gastroenterology 1987;93:719-26.

21. Kawasaki S, Imamura H, Bandai Y, Sanjo K, Idezuki Y. Direct evidence for the intact hepatocyte theory in patients with liver cirrhosis. Gastroenterology 1992;102:1351-5.

22. Meyer B, Luo HS, Bargetzi M, Renner EL, Stalder GA. Quantitation of intrinsic drug-metabolizing capacity in human liver biopsy specimens: support for the intact-hepatocyte theory. Hepatology 1991; 13:475-81

23. Sawamura T, Nakada H, Hazama H, Shiozaki Y, Sameshima Y, Tashiro Y. Hyperasialoglycoproteinemia in patients with chronic liver diseases and/or liver cell carcinoma. Asialoglycoprotein receptor in cirrhosis and liver cell carcinoma. Gastroenterology 1984;87:1217-21.

24. Yumoto Y, Umeda M, Ohshima K, Ogawa H, Kurokawa T, Kajitani M, Yumoto E, Hanafusa T, Tsuboi H, Higashi T. Estimation of remnant liver function before hepatectomy by means of technetium99m-diethylenetriamine-pentaacetic acid galactosyl human albumin. Cancer Chemother Pharmacol 1994;33 Suppl:S1-6.

25. Kudo M, Todo A, Ikekubo K, Hino M, Yonekura Y, Yamamoto $\mathrm{K}$, Torizuka K. Functional hepatic imaging with receptor-binding radiopharmaceutical: clinical potential as a measure of functioning hepatocyte mass. Gastroenterol Jpn 1991;26:734-41.

26. Kaneko M, Onda M, Tajiri T, Yoshida H, Kumita S, Kumazaki T. A new method of functional scintiphotosplenoportography using technetium-99m-DTPA-galactosylated human serum albumin. Hepatogastroenterology 2000;47:1636-41.

27. Yoshida H, Mamada Y, Taniai N, Hirakata A, Kawano Y, Kakinuma D, Mineta S, Tajiri T. Simultaneous evaluation of portal hemodynamics and liver function by scintiphotosplenoportography in pediatric recipients of living-donor liver transplants. Hepatogastroenterology 2009;56:819-23.

28. Mineta S, Yoshida H, Mamada Y, Taniai N, Mizuguchi Y, Akimaru K, Kumita S, Kumazaki T, Tajiri T. Changes in distribution of splenic venous flow in the patients with cirrhotic liver. Hepatogastroenterology 2005;52:1313-9.

29. Mochizuki K, Takatsuki M, Soyama A, Hidaka M, Obatake M, 
Eguchi S. The usefulness of a high-speed 3D-image analysis system in pediatric living donor liver transplantation. Ann Transplant 2012;17:31-4.

30. Kadokami T, Ando S, Momii H, Yoshida M, Narita S, Fukunaga T, Nishi J, Tamura A. Diagnostic performance of cardiac fusion images from myocardial perfusion imaging and multislice computed tomography coronary angiography for assessment of hemodynamically significant coronary artery lesions: an observational study. Nucl Med Commun 2012;33:60-8.
31. Yamashita K, Yoshiura T, Hiwatashi A, Tuvshinjargal D, Kamano $\mathrm{H}$, Shiratsuchi $\mathrm{H}$, Honda $\mathrm{H}$. Contributing factors in the pathogenesis of acquired chole3steatoma: size analysis based on MDCT. AJR Am J Roentgenol 2011;196:1172-5.

32. Komai Y, Sakai Y, Gotohda N, Kobayashi T, Kawakami S, Saito N. A novel 3-dimensional image analysis system for case-specific kidney anatomy and surgical simulation to facilitate clampless partial nephrectomy. Urology 2014;83:500-6. 